\title{
Dust Mass Concentration Measurement Based on Light Scattering Principle
}

\author{
Róbert Szabó* , Miroslav Badida \\ Technical University of Košice, Faculty of Mechanical Engineering, Letná 9, 04200 Košice
}

\section{BIOGRAPHICAL NOTES}

Róber Szabó, Ing. PhD., Received M.S. degree in mechanical engineering from Technical University of Košice, Slovakia in 1980 and Ph.D. degree in Environmental Engineering from Technical University of Košice, Slovakia in 2012. His research interests include environmental systems, dust mass concentration measurement and measurement of non-electric quantities. He was a main scientist research in several scientific projects. Now he is as general director of Section of science and technique at the Ministry of education, science, research and sport.

Dr.h.c. prof. Ing. Miroslav Badida, PhD., He is graduate of Mechanical Faculty of Technical University of Košice. His scientific and research work focuses on the field of environmental engineering. An accent is put on the issue of environmental management systems, ecologization of products and their production and life cycle analysis of products. Lately his attention is paid on research in the field of physical factors of working and living environment.

\section{KEY WORDS}

Dust, mass concentration, measurement, light scattering.

\section{ABSTRACT}

Paper deals with dust mass concentration measurement via using light scattering principle. This method enables continual monitoring of dust mass concentration. It is possible to identify leaks of dust and damage of filters. Light scattering principle depends on shape and color of dust particle. For this reason it is necessary to calibrate directly on measurement place, where is dust source located.

\section{Introduction}

One of the most monitored working area parameters, which can be dangerous for human health, is the dust mass concentration in environment air. From hygienic viewpoint, the dust is defined as small particles of solid state materials, which are dispersed in environment air otherwise which are sedimentary on various objects. These particles come from the various technological operations (metallurgical technological operations, combustion of various substances, ore-working industry, threadmills, cement industry, cereals cleaning, processing of dry vegetal materials, wood processing etc.).

Different physical phenomena are raised for different dust particles (gravity, air resistance, airflow, electrical attraction or repulsion). Particles with dimension over the $10 \mu \mathrm{m}$ are sedimentary in several minutes after their creation (or after repeatedly expelling) close to the dust source. Consequently, the working environment air is contaminated with particles smaller then $10 \mu \mathrm{m}$. Particles smaller then $10 \mu \mathrm{m}$ sedimentary 
very slowly and particles smaller then $0,1 \mu \mathrm{m}$ almost never sedimentary. So, every particles bigger than $10 \mu \mathrm{m}$ are very dangerous for human, because of their possibility to reach deeply inside the lungs $[1,2,3]$.

There is a variety of direct-reading aerosol monitors using light-scattering detectors (Fig. 1). These instruments generally have advantages in reduced weight, improved ruggedness, and continuous readout when compared with other direct-reading aerosol monitors. These instruments can be used to provide accurate dust concentration measurements as described below, though in most situations they are most useful for approximate or relative concentration measurements. Their principle advantage is that of providing real time information.

An aerosol is a group of particles suspended in the air. Aerosols can be introduced into the body primarily through the respiratory system. Total dust measurements indicate concentrations that can enter the nose and mouth of a worker as well as that which can settle on the skin while the respirable fraction of dust $[1,12]$

Light-scattering aerosol monitors (also called nephelometers or aerosol photometers) operate by illuminating aerosol passing through a defined volume and detecting the total light scattered by all the particles in that volume (Figure 1). Detection of the scattered light intensity permits the measurement of low dust concentrations in air. Its practical uses include flue gas measurement, determination of visibility in road tunnels, and the monitoring of filter installations for pollutants (insecticides, dyes, chemicals) coming from manufacturing processes. Aerosols of liquids such as sulfuric acid mist in exhaust gases and oil mist in ship engine rooms are also measured this way. [4].

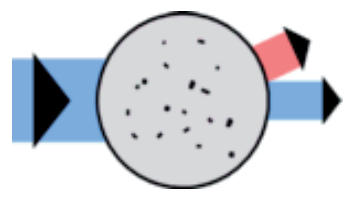

Fig. 1: Dust measurement at a scatter angle of $15^{\circ}$ [4].

\section{Scattered Light}

In an optically homogeneous medium (with constant refractive index and absorption), the light progresses in a straight line. Strictly speaking, this is the case only in a vacuum. Any change in the optical properties will deflect the light beam from its path. This physical process, which is referred to as the scattering of light by particles, causes the phenomenon of turbidity (Fig. 2).

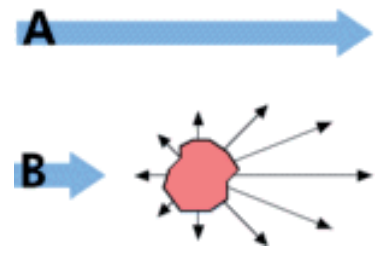

Fig. 2: Light propagation in a homogeneous medium (A) and in the presence of an obstacle (B) $[3,4]$.

The phenomenon is not limited to particles visible to the naked eye or in a microscope, however. Even in pure air or pure water, some scatter occurs at the molecules. Though this molecular scatter is very $p$, it can never be neglected entirely: for example, the sky looks blue because the sunlight is being scattered by the air's molecules.

One process that takes place when light is scattered is diffraction, and another is the excitation of radiation. Diffraction occurs because of the light's wave character: if a wave passes an obstacle in the immediate vicinity, it will be deflected from its path. The deflection angle depends on the relation between the wavelength and the size of the obstacle. The second process occurs because the atoms are excited, i.e. raised to higher energy levels, to radiate off the light that has struck them. This light will be radiated in various directions, depending on the particle characteristics, in accordance with the laws of light refraction, reflection, and dipole radiation. (A mirror's reflection is a special case of this.). The following Figure (fig. 3) illustrates the variables that affect the intensity of the scattered light $[3,4]$.

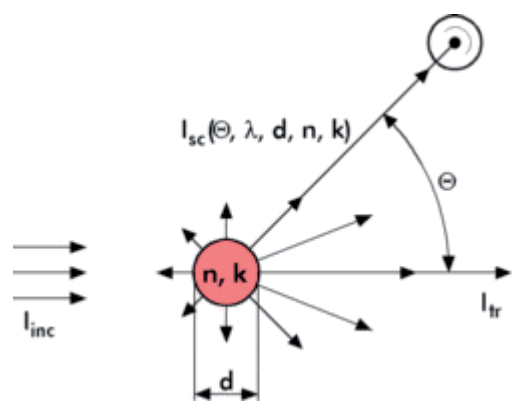

Fig. 3: Light scatter caused by a spheroidal particle [3, 4]. 
The diagram shows an incident light beam with intensity linc $I_{i n c}$ and wavelength $l$ striking a spheroidal particle. The intensity Isc of the scattered light is a function of the scatter angle, the particle size, the wavelength, and the optical properties of the particle and the medium.

Symbolically, then,

$$
I_{S C}=I_{S C}(Q, l, d, n)
$$

where $\mathrm{Q}$ represents the scatter angle, $\mathrm{d}$ the particle diameter, 1 the wavelength, and $n$ the refractive index in relation to the medium.

The explicit formulation of this equation is the subject of scatter theory. In the case of spheroidal particles, the general solution for any values of the variables is provided by the Mie Theory. It encompasses the Rayleigh Theory, which addresses the special case of very tiny particles $(<0.05 \mu \mathrm{m})$. The equation has a complex mathematical structure that lends itself to solution by computer.

A practical application of scattered light measurement is determination of the turbidity value, which provides information on the concentration of solids in liquid and gaseous media. The scatter angle is the angle between the scatter direction observed and the outlet axis of the transmitted

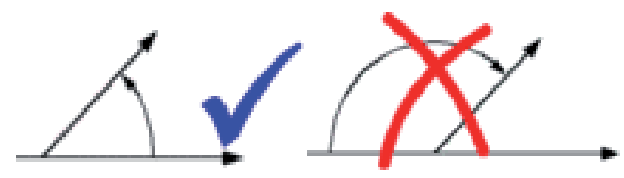

Fig. 4 Scatter angle $[3,4,5]$.

light (Fig. 4) $[3,4,5]$.

The polar diagram (Fig. 5) in the following Figure shows how the intensity of scatter varies as a function of the angle for two particle diameters.

As the diagram shows, very $\mathrm{p}$ particles scatter the light in all directions with about the same intensity. With increasing particle diameter, the scatter intensity increases generally and becomes lopsided, i.e. perceptibly greater at per angles.

This phenomenon is exploited by turbidimeter designers to improve their sensitivity: the per the measurement angle, the greater the amount of available light. But this relation is limited by the occurrence of stray light, which increases sharply

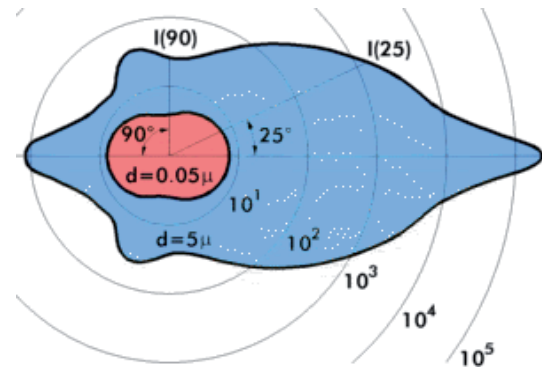

Fig. 5: Scattered light intensity as a function of angle for polydisperse suspensions of $\mathrm{SiO} 2$ in water. The total mass concentration is the same in both cases. Wavelength: $550 \mathrm{~nm}$. [3, 4, 5].

close to the transmitted light.

By measuring the scattered light at two different angles (such as $90^{\circ}$ and $25^{\circ}$ ), one can obtain a ratio to deduce the particle size. A practical application of such dual-angle measurement is the monitoring of beer filtration $[3,4,5]$.

\section{Particle Size and Color Influence to Dust Mass Concentration Measurement}

The light scattered by a particle and therefore the turbidity of a given suspension depends heavily on the size of the particle in question.

If the particle size in a suspension changes, either the number of particles or the aggregate particle mass may remain constant. The former situation (constant number of particles) occurs in the case of crystal growth or water buildup, the latter (constant aggregate mass) in the case of decomposition or agglomeration. This distinction is important, because the effects of the two cases on scattered light intensity are very different.

The following Figure (Fig. 6) illustrates how the scattered light intensity of various materials depends on particle diameter, assuming a constant number of particles. Above $0.3 \mu \mathrm{m}$, the scattered light increases in proportion to the square of the (effective) section of the particles. In the case of extremely p particles of less than $0.3 \mu \mathrm{m}$, the scattered light intensity increases in proportion to the square of the volume, i.e. raised to the sixth power of the diameter (dipole radiation) $[3,4,5]$.

Where the aggregate mass remains constant, the picture (Fig. 6) is entirely different. Above the maximum of $0.3 \mu \mathrm{m}$, the scattered light intensity declines in proportion to $1 / \mathrm{d}$ because the number of particles declines in proportion to the cube root even though their diameter increases in relation to 
the square. Below $0.3 \mu \mathrm{m}$, the scattered light inten-

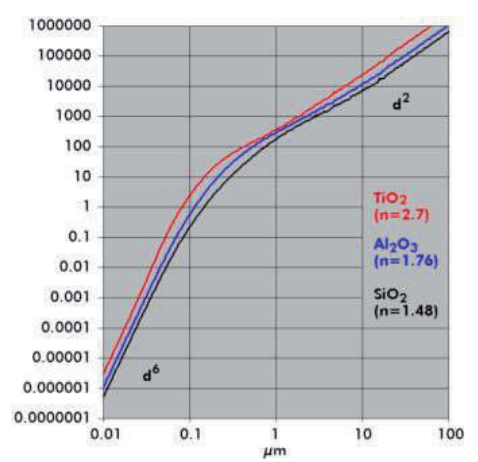

Fig. 6: Scattered light intensity for a constant number of particles. $n=$ refractive index, wavelength $550 \mathrm{~nm}, 90^{\circ}$ angle, particle size distribution: log. standard dist., $s=0.6 .[3,4,5]$

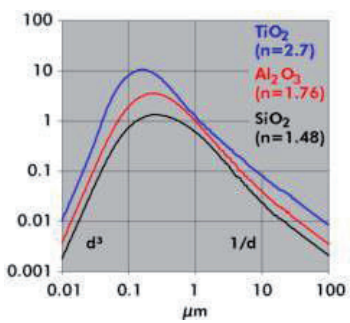

Fig. 7: Scattered light intensity for constant material volume $n=$ refractive index; wavelength $550 \mathrm{~nm} ; 90^{\circ}$ angle; particle size distribution: log. standard dist.; $s=0.6[3,4,5]$

sity increases in proportion to d cubed (Fig. 7).

In the real world, there is practically no such thing as a suspension with just one particle size (monodisperse). Normally one encounters a distribution of particle sizes (polydisperse). Nevertheless, the behavior remains essentially as depicted in the above graphs.

The effect of the particles' color on the intensity of the scattered light they create is generally overestimated. For example, people are often skeptical of the idea that even black particles will scatter light. It is an undisputed fact, however, that elementary carbon in various forms (soot, graphite, coal) produces scattered light of the same order of magnitude as white diatomaceous earth ( $\mathrm{SiO} 2)$. Dust measurement

The following Figure (Fig. 8) compares the scatter intensities of soot and white products of various particle size in air and water.

The Sensor FW100 (Fig. 9) series is designed to conduct continuous measurements of very low $\left(0.1 \mathrm{mg} / \mathrm{m}^{3}\right)$ to medium $\left(200 \mathrm{mg} / \mathrm{m}^{3}\right)$ dust concentrations - independent of the gas velocity, humidity, or charge of the particles involved. The probe design is particularly suitable for mounting on one side of the gas duct and does not re quire any mechanical adjustment or calibration with a dust-free measurement path. The measuring device can be used for a wide range of applications, including gas ducts with extremely small or large diameters,

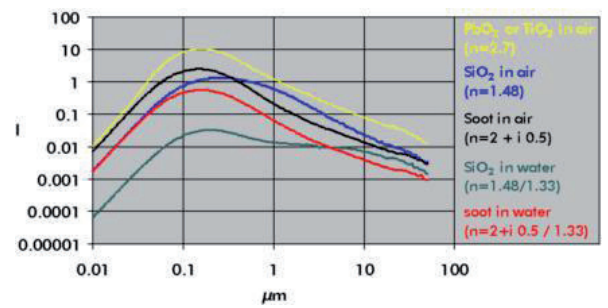

Fig. 8: Scatter intensity of various materials. Concentration $=1000$ ppm. $n=$ refractive index. [4]

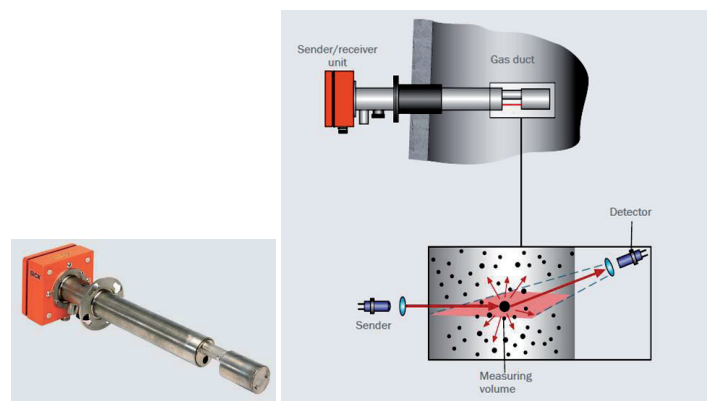

Fig. 9: Sensor FW100 - using Scattered Light Technology for the Monitoring of Dust Concentrations. [6]

as well as thick and thin-walled stacks.

The FW100 operates according to the scattered light measurement principle (forward scattering). Since it is extremely sensitive, this principle is particularly suitable for measuring very small particle concentrations. A laser diode directs a beam of modulated light (in the visible range) at the dust particles in the gas flow (Fig. 9). The light scattered by the particles is recorded by a highly sensitive detector. The point of intersection between the transmitted beam and the receiver aperture defines the measuring volume in the gas duct. The measured scattered light intensity is proportional to the dust concentration. After a gravimetric comparison measurement, for example according to EN 13284-1 and VDI 2066, therefore, a measurement signal that is directly proportional to the dust 
concentration can be output [6].

\section{Calibration of Dust Monitor Based on Light Scattering Principle}

Most photometers are factory-calibrated by comparing the instrument response in a welldefined aerosol to measurements by the gravimetric method (e.g. Method 0600). The instrument response at one or more concentrations is compared with the gravimetric method result. In most cases, the photometer response is modified to read directly in $\mathrm{mg} / \mathrm{m}^{3}$. However, it should be remembered that this calibration is only valid for the specific calibration aerosol and may differ by as much as a factor of ten when used with an aerosol from a different source, different composition, or size distribution. The factory calibration of this type is primarily useful to ensure that the instrument is operating properly and responding the same as other similar instruments. It does not ensure that the photometer will respond accurately to another aerosol. An aerosol photometer measures a single parameter that is dependent on many variables, e.g., particle size distribution, particle agglomeration, particle refractive index, that can and does change in field situations. Therefore, it is necessary that the aerosol photometer be calibrated in conditions closely approximating the aerosol to be monitored. Calibration is carried out by comparing the time-weighted average photometer readings directly with field measurements using Method 0600. Most photometers do not protect the optical surfaces with a clean air sheath and should be checked frequently for zero drift during calibration and routine monitoring [7-11].

\section{Conclusion}

Dust mass concentration measurement is very important quantity for evaluation of human environment. Dust particles cause many diseases and health problems. It is necessary to monitor environment for this purpose. As result of these measurements, it is possible to design any protection and devices for dust trapping. Photometer dust mass concentration measurements with fast, simple and capable of continuous measurement characteristics, is a widely used dust mass concentration measurement method. Our work is to study and develop a set of dust mass concentration mea- surement system based on the photometry [7-11].

\section{Acknowledge}

This contribution is also the result of the project implementation: Centre for research of control of technical, environmental and human risks for permanent development of production and products in mechanical engineering (ITMS:26220120060) supported by the Research \& Development Operational Programme funded by the ERDF and KEGA 049TUKE-4/2012.

\section{References}

[1] Air Quality - Particle Size Fraction Definitions for Health Related Sampling, Technical Report ISO/TR 7708-1983(E), International Standards Organization, Geneva, Switzerland (1983).

[2] Gmiterko, A., Slosarčík, S., Dovica, M.: Algorithm of Nonrespirable Dust Fraction Suppression Using an Optical Transducer of Dust Mass Concentration. IEEE Transactions on Instrumentation and Measurement. 47, 1998, No. 5, pp. 1228-1233.

[3] Kelemen, M., Matašovská, T.: Measurement of respirable fraction of dust mass concentration based on light scattering principle. In: Automa. Vol. 11, No. 5 (2005), pp. 20-23. - ISSN 1210-9592.

[4] Sigrist process photometer Glossary. Sigrist-Photometer AG. online. cited 2004-01-21. Last update: 2004-01-15. <http://www.photometer.com /en/abc.default.htm>

[5] Szabo, R:: Methodics of dust mass concentration measurement and balance of the dust emission in air. PhD thesis. Technical University of Kosice, Fac. Of Mech. Eng., Kosice, 2011.

[6] SICK MAIHAK GmbH. FW100 - using Scattered Light Technology for the Monitoring of Dust Concentrations. Available online. Cited 07-15-2013. Product information. http:// www.sick-maihak.ru/files/po_FW100_en.pdf

[7] Edvardsson, K., Magnusson, R.: Monitoring of dust emission on gravel roads: Development of a mobile methodology and examination of horizontal diffusion Original Research Article. Atmospheric Environment, Volume 43, Issue 4, February 2009, Pages 889-896

[8] Etyemezian, V., Kuhns, H., Gillies, J., Green, M., Pitchford, M., Watson, J.: Vehicle-based road dust emission measurement: 1 -methods and calibration Original Research Article. Atmospheric Environment, Volume 37, Issue 32, October 2003, Pages 4559-4571.

[9] Shin-Fu Chiou, Chuen-Jinn Tsai: Measurement of emission factor of road dust in a wind tunnel Original Research Article. Powder Technology, Volume 118, Issues 1-2, 8 August 
Acta Mechanica Slovaca

Journal published by Faculty of Mechanical Engineering - Technical University of Košice

2001, Pages 10-15

[10] R.-M. Hu, R.S. Sokhi: Light scattering and absorption properties of dust particles retrieved from satellite measurements Original Research Article. Journal of Quantitative Spectroscopy and Radiative Transfer, Volume 110, Issues 14-16, September-November 2009, Pages 1698-1705.

[11] Siew Lai Huang, Chun-Yang Yin, Siaw Yang Yap: Particle size and metals concentrations of dust from a paint manufacturing plant Original Research Article. Journal of Hazardous Materials, Volume 174, Issues 1-3, 15 February 2010, Pages 839-842

[12] Petrovová, E., Maženský, D., Luptáková, L., Holovská, K., Špaleková, E., Massanyi, P., Haladová, E., Tóth, T.: Alterations in the rabbit lymphoid tissue after bendiocarb administration, Journal of Environmental Science and Health : Part B : Pesticides, Food Contaminants, and Agricultural Wastes. Vol. 45, no. 7, 2010, p. 719-728., ISSN 0360-1234 